\title{
Publisher's Note: Improved empirical parameterization for projectile fragmentation cross sections [Phys. Rev. C 95, 034608 (2017)]
}

\author{
B. Mei
}

(Received 29 March 2017; published 5 April 2017)

DOI: 10.1103/PhysRevC.95.049902

This paper was published online on 17 March 2017 with errors in the caption of Fig. 6 and in the text on page 7. The first sentence of the caption to Fig. 6 should read as "Experimental cross sections of fragments along a constant $T_{z}$ chain from $T_{z}=-1 / 2$ to $T_{z}=+1$, produced in the projectile fragmentation of ${ }^{58} \mathrm{Ni}$ on ${ }^{181} \mathrm{Ta}$ targets at $140 \mathrm{MeV} /$ nucleon [16] (filled squares joined by full lines) and ${ }^{9} \mathrm{Be}$ targets at $650 \mathrm{MeV} /$ nucleon [5] (open squares joined by full lines)." The last sentence in the left-hand column on page 7 should read as "These cross sections along a constant $T_{z}$ chain measured in the fragmentation of ${ }^{58} \mathrm{Ni}$ on ${ }^{181} \mathrm{Ta}$ targets at $140 \mathrm{MeV} /$ nucleon (filled squares) [16] and ${ }^{9} \mathrm{Be}$ targets at $650 \mathrm{MeV} /$ nucleon (open squares) [5] are compared with predictions by FRACS and EPAX3 in Fig. 6." The paper has been corrected as of 30 March 2017. The caption and text are correct in the printed version of the journal. 